\title{
A method employing STM for the estimation of relative changes in the work function of modified metal tips
}

\author{
R B SHARMA ${ }^{\dagger}$, C P VINOD and G U KULKARNI* \\ Chemistry and Physics of Materials Unit, Jawaharlal Nehru Centre for Advanced Scientific Research, Jakkur P.O., \\ Bangalore 560 064, India \\ ${ }^{\dagger}$ INS Shivaji, Lonavla 410 402, India
}

MS received 22 May 2001; revised 3 May 2002

\begin{abstract}
I-z$ spectroscopy measurements using a scanning tunnelling microscope (STM) were carried out to determine the change in the work function of a $\mathrm{W}$ tip following one monolayer (1 ML) deposition of Ni and subsequent annealing at $700 \mathrm{~K}$. The variation in the actual gap voltage obtained from the $I-z$ data of the clean tip was used in the calculation. The estimated values of the change in work function, $0 \cdot 16 \mathrm{eV}$ and $0.59 \mathrm{eV}$, for as-deposited and annealed tips, respectively match closely with the reported values. The method is generally applicable to chemically modified metal tips.
\end{abstract}

Keywords. I-z spectroscopy; scanning tunneling microscope; workfunction.

\section{Introduction}

Surface modification at nanoscale of metals and semiconductors is important for a variety of applications in the field of microelectronics. Field emission devices for example, employ tips that have been subjected to physical and chemical procedures to improve the performance. Local properties such as work function, change following such modifications and it is indeed crucial to monitor and control the changes in order to arrive at desired properties. Field emission microscope (FEM) has been used in many cases to detect changes in the work function of a variety of metal tips following gas adsorption (Gomer 1961) and metal deposition (Joag and Jones 1984; Sidorski 1984; Gotoh et al 1996; Nagao et al 1996; Sharma et al 1998). This method uses high tip-anode voltages of the order of $\mathrm{kV}$ to achieve electron emission. The current trend in technology, however, demands the measurements to be carried out at low voltages.

We have explored the possibility of determining the changes in the work function following tip modification by tunneling measurements under low voltages using a STM set up. STM offers a high-resolution probe to measure local work function variations caused by subtle changes of the tip surface. The experiment involves measurement of the tunnel current, $I$, as a function of the tip-sample distance, $z$, under a given bias voltage of $V$. The local work function $(\phi)$ is related to the gap conductance, $G$, as follows (Bonnell 1993)

$$
\phi=0.952 \times(\mathrm{d} \ln G / \mathrm{d} z)^{2} .
$$

\footnotetext{
*Author for correspondence
}

Studies using STM mostly concentrate on work function measurements of metal surfaces. Jia et al (1998) determined the variations in the local work function at the steps on $\mathrm{Cu}(111)$ and $\mathrm{Au}(111)$ surfaces. Clarke et al (1996) studied the tip-surface interactions in the case of $\mathrm{W}$ tip/Cu(100) surface. Using a W tip, Olesen et al (1996) measured the apparent barrier height at $\mathrm{Au}(110)$, $\mathrm{Pt}(100)$ and $\mathrm{Ni}(100)$ surfaces by measuring the tunnel current and voltage simultaneously. They showed that for a reliable estimation of the gap conductance from an $I-Z$ measurement, it is necessary to consider the variations in the gap voltage as well.

We have found an alternative procedure to measure the relative changes in the work function of a metal tip employing STM in the default d.c. mode. The $V-z$ variations are derived using (1), based on the known work function of the clean tip

$$
(\mathrm{d} \ln V / \mathrm{d} z)_{\text {clean }}=(\mathrm{d} \ln I / \mathrm{d} z)_{\text {clean }}+\sqrt{ } \phi_{\text {clean }} .
$$

The gap conductance after tip-modification is estimated assuming that the circuit characteristics remain similar.

$$
(\mathrm{d} \ln G / \mathrm{d} z)_{\text {modified }}=(\mathrm{d} \ln I / \mathrm{d} z)_{\text {modified }}-(\mathrm{d} \ln V / \mathrm{d} z)_{\text {clean }} .
$$

The relative change in the work function is then expressed as

$$
\Delta \phi=\phi_{\text {modified }}-\phi_{\text {clean }} .
$$

This method was tested using a Ni modified $\mathrm{W}$ tip in a UHV STM set up. The measured values of the work function of as-deposited and annealed tips have been found to be in agreement with the reported ones. 


\section{Experimental}

Scanning tunnelling measurements were carried out on a UHV STM Omicron Vakuumphysik instrument. The W tips were prepared by electrochemical etching and were stored in UHV. A freshly cleaved highly oriented pyrolytic graphite (HOPG) surface was used as the substrate. The STM images of the graphite were first obtained to check the quality of the tips. For the spectroscopic measurements, the tip was positioned on a large flat terrace on the substrate away from the steps. A constant bias voltage of $91 \mathrm{mV}$ was applied to the sample with the tip at ground potential. A set current $\left(I_{\text {set }}\right)$ of $10 \mathrm{nA}$ was used. The feedback loop was temporarily disabled and a voltage ramp was superimposed on the reference of the feedback for achieving a controlled movement of the tip along the $z$-axis with respect to initial position $(z=0)$. Initially, the tip was moved by $9 \AA$ towards the surface $(z=-9 \AA)$ and then the variations in the tunnel current, $I$, were recorded while retracting from the substrate with $z$ varying from $-9 \AA$ to $+10 \AA$ at 200 data points with an acquisition time of $400 \mu \mathrm{s}$ at each point and a delay of $2000 \mu$ s between two consecutive measurements. The speed of the tip was set at $500 \mathrm{~nm} / \mathrm{s}$.

The $I-z$ measurements were first performed with a clean $\mathrm{W}$ tip in UHV. In situ deposition of high purity
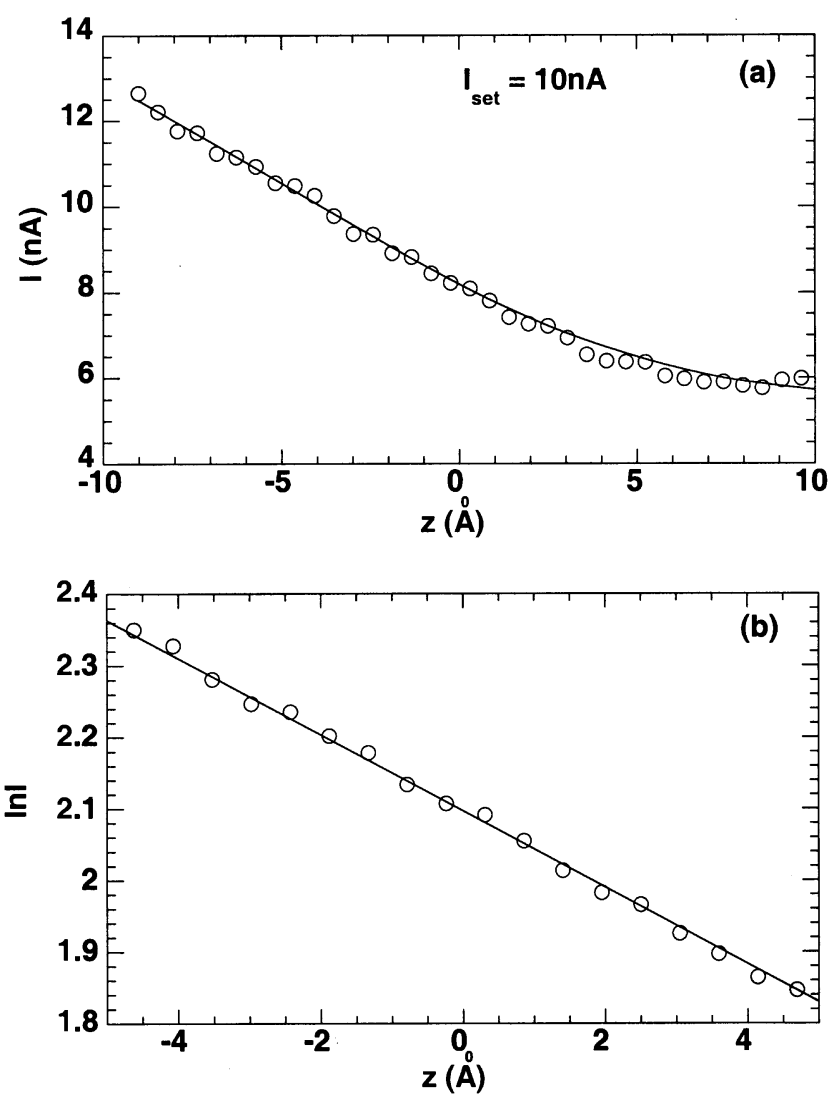

Figure 1. (a) Variation in the tunnel current, I, with tipsample separation, $z$ and (b) that of $\ln I$ with $z$. nickel (99.99\%, Aldrich) on W tip was done by resistive evaporation. A coverage of $\sim 1$ monolayer was obtained based on a calibration done using XPS measurements on a graphite surface with an identical Ni coverage (Seah and Dench 1979). The $I-z$ spectroscopy measurements were repeated on the as-deposited tip. The tip was then annealed in UHV at $700 \mathrm{~K}$ for $300 \mathrm{~s}$. The quality of the HOPG image was also improved following annealing. $I-z$ measurements were then performed on the annealed tip under similar conditions.

\section{Results and discussion}

Figure 1a shows the $I-z$ characteristics of a clean $W$ tip obtained with a gap voltage of $91 \mathrm{mV}$ and a set current $\left(I_{\text {set }}\right)$ of $10 \mathrm{nA}$. In the advanced position $(z=-9 \AA)$, the tunnel current is $\sim 13 \mathrm{nA}$ and it decreases rapidly to $6 \mathrm{nA}$ in the retracted mode at $z=+10 \AA$. We notice that while passing through initial position $(z=0)$, the current is slightly less $(\sim 8 \mathrm{nA})$ than the $I_{\text {set }}$. In order to extract the actual gap voltage variations during the $I-z$ measurement, we calculated $\ln I$ for various $z$ values (figure 1b). Assuming that $\phi_{\mathrm{W}}=4.54 \mathrm{eV}$, we obtained $(\mathrm{d} \ln V / \mathrm{d} z)_{\text {clean }}$ from the slope of the linear fit shown in figure $1 b$ using (2).
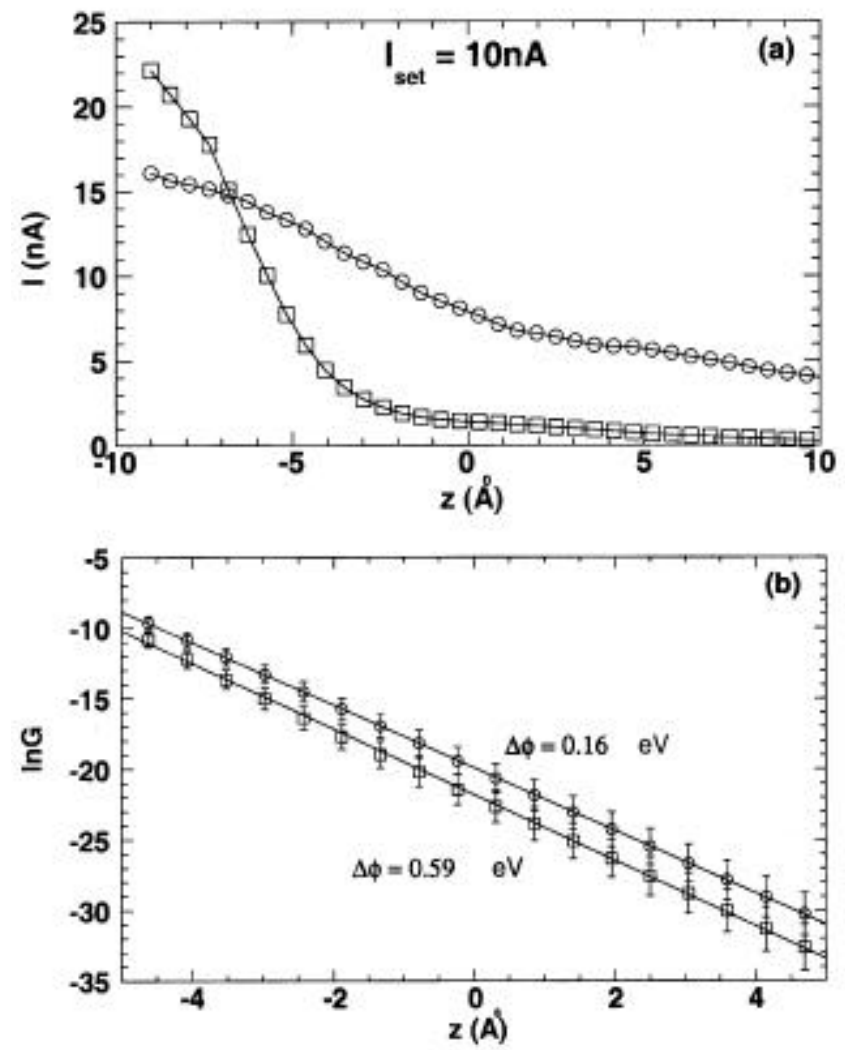

Figure 2. Variations in the tunnel current, $I,(\mathbf{a})$ and $\ln G(\mathbf{b})$ with tip-sample separation, $z$; as-deposited, circles; annealed, squares. 
In figure $2 \mathrm{a}$, we show the $I-z$ characteristics for the deposited and annealed tips. We observe that in the case of as-deposited tip, the current starts from $\sim 16 \mathrm{nA}$ at $z=-9 \AA$ and decreases gradually to a value of $4 \mathrm{nA}$ at $z$ $=10 \AA$ while in case of the annealed tip, the current starts at $\sim 22 \mathrm{nA}$ and decreases rapidly to zero at higher distances. The enhancement in the tunnel current arises due to the new states near tip apex (Bonnell 1993).

The value of $(\mathrm{d} \ln I / \mathrm{d} z)_{\text {modified }}$, obtained from the respective $I-z$ characteristics was substituted in (3) to calculate $(\mathrm{d} \ln G / \mathrm{d} z)_{\text {modified }}$. This provided the estimation of the work function of the modified tip. However, the values obtained rely on the conditions employed for the clean tip and therefore, only the relative change and not the absolute value of the work function is to be taken seriously. Using (4), we obtained $\Delta \phi$ of +0.16 and $+0.59 \mathrm{eV}$ for as-deposited and annealed tips, respectively. In order to validate our method, we calculated $\ln G$ values from $(\mathrm{d} \ln I / \mathrm{d} z)_{\text {modified }}$ in both the cases and obtained linear variations with $z$ as expected (Bonnell 1993; Olesen et al 1996) (see figure 2b).

An earlier study from this laboratory using XPS measurements (Kulkarni and Vinod 1997) has shown that the binding energy of $2 p_{3 / 2}$ core-level of nickel deposited on a polycrystalline tungsten surface shifts by $\sim+0.15 \mathrm{eV}$ for a metal coverage of one monolayer. This enhancement was attributed to a charge transfer process across the $\mathrm{Ni} / \mathrm{W}$ interface leading to an increase in the work function. The present finding of the change in the work function of the as-deposited tip agrees well with the XPS study. Also, the change in the work function obtained in the case of annealed tip $(+0.59 \mathrm{eV})$ is quite comparable with that from a field emission study $(+0.52 \mathrm{eV})$ (Sharma et al 1998).

We have used several sets of bias voltage and set current in order to ascertain their influence on the work function measurements. For lower value of $I_{\text {set }}(\sim 1 \mathrm{nA})$, the estimated values of the work function were found to be similar to that at $10 \mathrm{nA}$. At higher $I_{\text {set }}$ value of $20 \mathrm{nA}$, the values were significantly lower, probably due to induced polarization at relatively smaller tip-sample separations. However, the measurements were not very sensitive to the changes in the bias voltages.

\section{Acknowledgement}

The authors thank Prof. C N R Rao, FRS, for helpful discussions and encouragement.

\section{References}

Bonnell D A 1993 Scanning tunneling microscopy and spectroscopy. Theory, techniques and applications (New York: VCH)

Clarke A R H, Pethica J B, Nieminen J A, Basenbacher F, Laegsgaard E and Stensgaard I 1996 Phys. Rev. Lett. 761276

Gomer R 1961 Field emission and field ionization (Harvard, Cambridge, MA: Harvard University Press)

Gotoh Y, Nagao M, Matsubara M, Inoue K, Tsuji H and Ishikawa J 1996 Jpn J. Appl. Phys. 35 L1297

Jia J F, Inoue K, Hasegawa Y, Yang W S and Sakurai T 1998 Phys. Rev. $\mathbf{B 5 8} 1193$

Joag D S and Jones J P 1984 J. Phys. (Paris) C9 59

Kulkarni G U and Vinod C P 1997 Appl. Surf. Sci. 115336

Nagao M, Matsubara M, Inoue K, Gotoh Y, Tsuji H and Ishikawa J 1996 Jpn J. Appl. Phys. 355479

Olesen L, Brandbyge M, Sorensen M R, Jacobsen K W, Laegsgaard E, Stensgaard I and Besenbacher F 1996 Phys. Rev. Lett. 761485

Seah M P and Dench W A 1979 Surf. Interface. Anal. 12

Sharma R B, Yawalkar P W, Pradeep N and Joag D S 1998 Ultramicroscopy $\mathbf{7 3} 99$

Sidorski Z 1984 Appl. Phys. A33 213 